\title{
EXISTENCE THEOREM FOR NONCONVEX STOCHASTIC INCLUSIONS
}

\author{
MICHA£ KISIELEWICZ \\ Higher College of Engineering \\ Institute of Mathematics \\ Podgórna 50, 65-246 Zielona Góra, POLAND
}

(Received November, 1993; revised March, 1994)

\begin{abstract}
An existence theorem for stochastic inclusions $x_{t}-x_{s} \in \int_{s}^{t} F_{\tau}\left(x_{\tau}\right) d \tau$ $+\int_{s}^{t} G_{\tau}\left(x_{\tau}\right) d w_{\tau}+\int_{s}^{t} \int_{\mathbb{R}^{n}} H_{\tau, z}\left(x_{\tau}\right) \tilde{\nu}(d \tau, d z)$ with nonanticipative nonconvexvalued right-hand sides is proved.
\end{abstract}

Key words: Stochastic inclusions, existence solutions, solution set.

AMS (MOS) subject classifications: 93E03, 93C30.

\section{Introduction}

Existence theorem and weak compactness of the solution set to stochastic inclusion

$$
x_{t}-x_{s} \in \int_{s}^{t} F_{\tau}\left(x_{\tau}\right) d \tau+\int_{s}^{t} G_{\tau}\left(x_{\tau}\right) d w_{\tau}+\int_{s}^{t} \int_{\mathbb{R}^{n}} H_{\tau, z}\left(x_{\tau}\right) \tilde{\nu}(d \tau, d z),
$$

denoted by $S I(F, G, H)$, with predictable convex-valued right-hand sides have been considered in the author's paper [4]. These results were obtained by fixed points methods. Applying the successive approximation method we shall prove here an existence theorem for $S I(F, G, H)$ with nonanticipative nonconvex-valued multivalued processes $F, G$ and $H$. To begin with, we recall the basic definitions dealing with set-valued stochastic integrals and stochastic inclusions presented in [5].

Let a complete filtered probability space $\left(\Omega, \mathscr{F}_{,}\left(\mathscr{F}_{t}\right)_{t}>0, P\right)$ be given, where a family $\left(\mathscr{F}_{t}\right)_{t \geq 0}$,

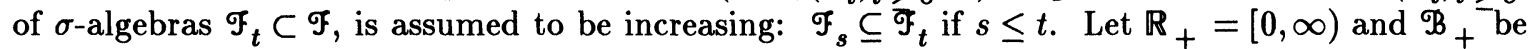
the Borel $\sigma$-algebra on $\mathbb{R}_{+}$. We consider set-valued stochastic processes $\left(\mathscr{F}_{t}\right)_{t \geq 0},\left(\mathscr{G}_{t}\right)_{t \geq 0}$ and

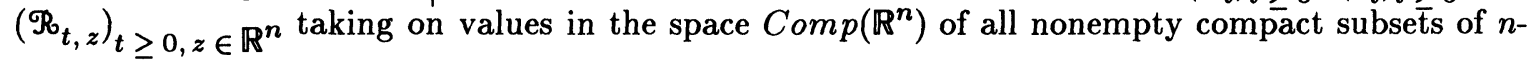
dimensional Euclidean space $\mathbb{R}^{n}$. They are assumed to be nonanticipative and such that $\int_{0}^{\infty}\left\|\mathscr{F}_{t}\right\|^{p} d t<\infty, \quad p \geq 1, \int_{0}^{\infty}\left\|\mathcal{G}_{t}\right\|^{2} d t<\infty$ and $\int_{0}^{\infty} \int_{\mathbb{R}^{n}}\left\|\mathscr{R}_{t, z}\right\|^{2} d t q(d z)<\infty$, a.s., where $q$ is a measure on a Borel $\sigma$-algebra $\mathscr{B}^{n}$ of $\mathbb{R}^{n}$ and $\|A\|:=\sup \{|a|: a \in A\}, A \in \operatorname{Comp}\left(\mathbb{R}^{n}\right)$. The space $\operatorname{Comp}\left(\mathbb{R}^{n}\right)$ is considered with the Hausdorff metric $h$ defined in the usual way, i.e., $h(A, B)=\max \{\bar{h}(A, B), \bar{h}(B, A)\}$, for $A, B \in \operatorname{Comp}\left(\mathbb{R}^{N}\right)$, where $\bar{h}(A, B)=\{\operatorname{dist}(a, B): a \in A\}$ and $\bar{h}(B, A)=\{\operatorname{dist}(b, A): b \in B\}$. 


\section{Basic Definitions and Notations}

Throughout the paper, we shall assume that a filtered complete probability space $\left(\Omega, \mathscr{F}_{,}\left(\mathscr{F}_{t}\right)_{t>0}, P\right)$ satisfies the following usual hypotheses:

(i) $\quad \mathcal{F}_{0}$ contains all the $P$-null sets of $\mathscr{F}$ and

(ii) $\mathscr{F}_{t}=\bigcup_{u>t} \mathscr{F}_{u}$, all $t, \quad 0 \leq t<\infty$; that is, the filtration $\left(\mathscr{F}_{t}\right)_{t \geq 0}$ is right continuous.

As usual, we shall consider a set $\mathbb{R}_{+} \times \Omega$ as a measurable space with the product $\sigma$-algebra $\mathscr{B}+\otimes \mathscr{F}$.

An $n$-dimensional stochastic process $x$ is understood as a function $x: \mathbb{R}+\times \Omega \rightarrow \mathbb{R}^{n}$ with $\mathscr{F}$ measurable sections $x_{t}$, for $t \geq 0$, and it is denoted by $\left(x_{t}\right)_{t \geq 0}$. It is measurable if $x$ is $\mathfrak{B}_{+} \otimes \mathcal{F}_{-}$ measurable. The process $\left(x_{t}\right)_{t \geq 0}$ is $\mathscr{F}_{t}$-adapted or adapted if $x_{t}$ is $\mathscr{F}_{t}$-measurable for $t \geq 0$. Every measurable and adapted process is called nonanticipative. In what follows, the Banach spaces $L^{p}\left(\Omega, \mathscr{F}_{t}, P, \mathbb{R}^{n}\right)$ and $L^{p}\left(\Omega, \mathcal{F}, P, \mathbb{R}^{n}\right)$ with the usual norm $\|\cdot\|$ are denoted by $L_{n}^{p}\left(\mathscr{F}_{t}\right)$ and $L_{n}^{p}(\mathscr{F})$, respectively.

Let $\mathcal{H}^{2}\left(\mathscr{F}_{t}\right)$ denote the family of all (equivalence classes of) $n$-dimensional nonanticipative processes $\left(f_{t}\right)_{t \geq 0}$ such that $\int_{0}^{\infty}\left|f_{t}\right|^{2} d t<\infty$, a.s. We shall also consider a subspace $\ell_{n}^{2}$ of $\mathcal{H}^{2}\left(\mathcal{F}_{t}\right)$ defined by $\mathcal{L}_{n}^{2}=\left\{\left(f_{t}\right)_{t \geq 0} \in \mathcal{H}^{2}\left(\mathcal{F}_{t}\right): E \int_{0}^{\infty}\left|f_{t}\right|^{2} d t<\infty\right\}$ with the norm $\|\cdot\|_{\ell_{n}^{2}}$ defined in the usual way. The Banach spaces $L^{p}\left(\mathbb{R}_{+}, \mathscr{B}_{+}, d t, \mathbb{R}_{+}\right), p \geq 1$ and $L^{2}\left(\mathbb{R}_{+} \times \mathbb{R}^{n}, \mathscr{B}_{+} \otimes \mathscr{B}^{n}, d t \times q, \mathbb{R}_{+}\right)$, with the usual norms $|\cdot|_{p}$ and $\|\cdot\|_{2}$ will be denoted by $L^{p}\left(\mathscr{B}_{+}^{+}\right)$and $L^{2}\left(\mathscr{B}_{+} \times \mathscr{B}^{n}\right)$, respectively. Finally, by $M_{n}\left(\mathscr{F}_{t}\right)$ we denote a space of all (equivalence classes of) $n$-dimensional $\mathcal{F}_{t}$-measurable mappings.

Throughout the paper, by $\left(w_{t}\right)_{t} \geq 0$ we mean a one-dimensional $\mathscr{F}_{t}$-Brownian motion starting at 0 , i.e., such that $P\left(w_{0}=0\right)=1$. By $\nu(t, A)$ we denote a $\mathscr{F}_{t}$-Poisson measure (see [1]) on $\mathbb{R}_{+} \times \mathscr{P}^{n}$ and then define an $\mathscr{F}_{t}$-centered Poisson measure $\widetilde{\nu}(t, A), t \geq 0, A \in \mathscr{B}^{n}$, by taking $\widetilde{\nu}(t, A)=\nu(t, A)-t q(A), t \geq 0, A \in \mathscr{B}^{n}$, where $q$ is a measure on $\mathscr{B}^{n}$ such that $E \nu(t, B)=t q(B)$ and $q(B)<\infty$ for $B \in \mathfrak{B}_{0}^{n}$.

By $\mathcal{H}^{2}\left(\mathscr{F}_{t}, q\right)$, we shall denote the family of all (equivalence classes of) $\mathfrak{B}_{+} \otimes \mathscr{F} \otimes \mathfrak{B}^{n}-$

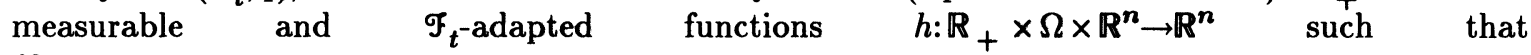
$\int_{0}^{\infty} \int_{\mathbb{R}^{n}}\left|h_{t, z}\right|^{2} d t q(d z)<\infty$ a.s. Recall that a function $h: \mathbb{R}_{+} \times \Omega \times \mathbb{R}^{n} \rightarrow \mathbb{R}^{n}$ is said to be $\mathscr{F}_{t}$-adapted or adapted if for every $x \in \mathbb{R}^{n}$ and $t \geq 0, h(t, \cdot, x)$ is $\mathscr{F}_{t}$-measurable.

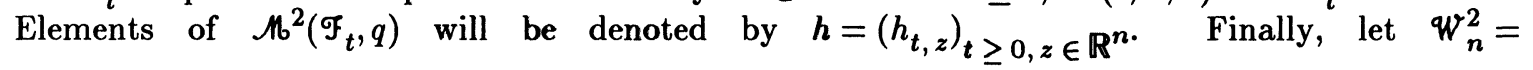
$\left\{h \in \mathcal{M}^{2}\left(\mathcal{F}_{t}, q\right):\|h\|_{W_{n}^{2}}^{2}<\infty\right\}$ where $\|h\|_{W_{n}^{2}}^{2}=E \int_{0}^{\infty} \int_{\mathbb{R}^{n}}\left|h_{t, z}\right|^{2} d t q(d z)$.

$\begin{array}{ccccc}\text { Given } \quad g \in \mathcal{M}^{2}\left(\mathscr{F}_{t}\right) & \text { and } & h \in \mathcal{M}^{2}\left(\mathscr{F}_{t}, q\right), \quad \text { by } \quad\left(\int_{0}^{t} g_{\tau} d w_{\tau}\right)_{t \geq 0} \quad \text { and } \\ \left(\int_{0}^{t} \int_{\mathbb{R}^{n} h_{\tau, z}} \widetilde{\nu}(d \tau, d z)\right)_{t \geq 0}, & \text { we denote their stochastic integrals with respect to an } \mathscr{F}_{t^{-}}\end{array}$ Brownian motion $\left(w_{t}\right)_{t \geq 0}$ and an $\mathscr{F}_{t}$-centered Poisson measure $\tilde{\nu}(t, A), t \geq 0, A \in \mathfrak{B}^{n}$, respectively. These integrals, understood as $n$-dimensional stochastic processes, have quite similar properties (see [1]).

Let us denote by $D$ the family of all $n$-dimensional $\mathscr{F}_{t}$-adapted cádlág (see [6]) processes $\left(x_{t}\right)_{t \geq 0}$ such that Esup $t \geq 0\left|x_{t}\right|^{2}<\infty$. The space $D$ is considered as a normed space with the 
norm $\|\xi\|_{\ell}=\left\|\sup _{t \geq 0}\left|\xi_{t}\right|\right\|_{L^{2}}$ for $\xi=\left(\xi_{t}\right)_{t \geq 0} \in D$, where $\|\cdot\|_{L^{2}}$ is a norm of $L^{2}(\Omega, \mathcal{F}, P, \mathbb{R})$. It can be verified that $\left(D,\|\cdot\|_{\ell}\right)$ is a Banach space.

Given $0 \leq \alpha<\beta<\infty$ and $\left(x_{t}\right)_{t \geq 0} \in D$, let $x^{\alpha, \beta}=\left(x_{t}^{\alpha, \beta}\right)_{t \geq 0}$ be such that $x_{t}^{\alpha, \beta}=x_{\alpha}$ and $x_{t}^{\alpha, \beta}=x_{\beta}$ for $0 \leq t \leq \alpha$ and $t \geq \beta$, respectively, and $x_{t}^{\alpha, \beta}=x_{t}$ for $\alpha \leq t \leq \beta$. It is clear that

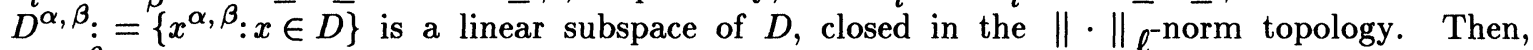
$\left(D^{\alpha, \beta},\|\cdot\|_{\ell}\right)$ is also a Banach space.

Given a measure space $(X, \mathfrak{B}, m)$, a set-valued function $\mathscr{B}: X \rightarrow C l\left(\mathbb{R}^{n}\right)$ is said to be $\mathscr{B}$ measurable if $\{x \in X: \mathscr{B}(x) \cap C \neq \emptyset\} \in \mathscr{B}$ for every closed set $C \subset \mathbb{R}^{n}$. For such a multifunction,

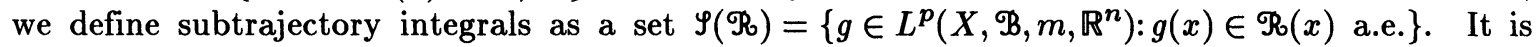
clear that for nonemptiness of $\varphi\left(\mathscr{B}_{0}\right)$ we must assume more then $\mathscr{B}$-measurability of $\mathscr{B}$. In what follows, we shall assume that $\mathscr{B}$-measurable set-valued function $\mathscr{B}_{B}: X \rightarrow C l\left(\mathbb{R}^{n}\right)$ is $p$-integrable bounded, $\quad p \geq 1$, i.e., that a real-valued mapping: $X \ni x \rightarrow\left\|\mathscr{R}_{0}(x)\right\| \in \mathbb{R}_{+}$belongs to $L^{p}\left(X, \mathfrak{B}, m, \mathbb{R}_{+}\right)$. It can be verified (see [2], Th. 3.2) that a $\mathfrak{B}$-measurable set-valued mapping $\mathscr{R}: X \rightarrow C l\left(\mathbb{R}^{n}\right)$ is $p$-integrable bounded, $p \geq 1$, if and only if $\varphi(\mathscr{R})$ is nonempty and bounded in $L^{p}\left(X, \mathfrak{B}, m, \mathbb{R}^{n}\right)$. Finally, it is easy to see that $\mathscr{\varphi}(\mathscr{B})$ is decomposable, i.e., such that

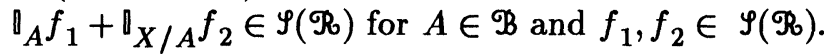

We have the following general result dealing with the properties of subtrajectory integrals (see [2], [3]).

Proposition 1. Let $\mathfrak{B}: X \rightarrow C l\left(\mathbb{R}^{n}\right)$ be $\mathfrak{B}$-measurable and $p$-integrable bounded, $p \geq 1$. Then, $\varphi(\mathscr{B})$ is a nonempty bounded and closed subset of $L^{p}\left(X, \mathscr{B}, m, \mathbb{R}^{n}\right)$. Moreover, if $\mathscr{B}$ takes on convex values then $\mathscr{Y}\left(\mathscr{R}_{B}\right)$ is convex and weakly compact in $L^{p}(X, \mathfrak{B}, m, \mathbb{R})$.

Let $\mathcal{G}=\left(\mathcal{G}_{t}\right)_{t>0}$ be a set-valued stochastic process with values in $C l\left(\mathbb{R}^{n}\right)$, i.e., a family of $\mathscr{F}$ measurable set-valued mappings $g_{t}: \Omega \rightarrow C l\left(\mathbb{R}^{n}\right), t \geq 0$. We call $\mathfrak{g}$ measurable if it is $\mathfrak{B}_{+} \otimes \mathcal{F}_{-}$ measurable. Similarly, $\mathcal{G}$ is said to be $\mathscr{F}_{t}$-adapted or adapted if $\mathcal{G}_{t}$ is $\mathscr{F}_{t}$-measurable for each $t \geq 0$. A measurable and adapted set-valued stochastic process is called nonanticipative.

In what follows, we shall also consider $\mathfrak{B}_{+} \otimes \mathscr{F} \otimes \mathfrak{B}^{n}$-measurable set-valued mappings $\mathscr{B}_{0}: \mathbb{R}_{+} \times \Omega \times \mathbb{R}^{n} \rightarrow C l\left(\mathbb{R}^{n}\right)$. They will be denoted as families $\left(\mathscr{R}_{t, z}\right)_{t \geq 0, z \in \mathbb{R}^{n} \text { and called }}$ measurable set-valued stochastic processes depending on a parameter $z \in \mathbb{R}^{n}$. The process $\mathscr{B}_{0}=\left(\mathscr{R}_{t, z}\right)_{t \geq 0, z \in \mathbb{R}^{n}}$ is said to be $\mathscr{F}_{t}$-adapted or adapted if $\mathscr{R}_{t, z}$ is $\mathscr{F}_{t}$-measurable for each $t \geq 0$ and $z \in \mathbb{R}^{n}$. We call it nonanticipative if it is measurable and adapted.

Denote by $\mathcal{M}_{s-v}^{2}\left(\mathscr{F}_{t}\right)$ and $\mathcal{M}_{s-v}^{2}\left(\mathscr{F}_{t}, q\right)$ families of all nonanticipative set-valued processes

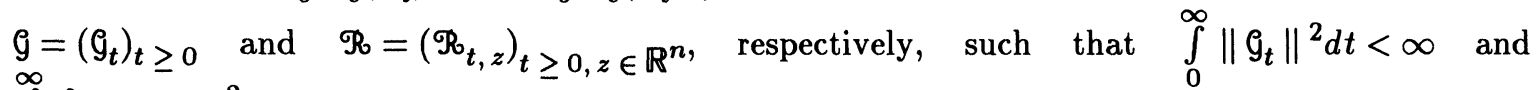
$\int_{0}^{\infty} \int_{\mathbb{R}^{n}}\left\|\mathfrak{R}_{t, z}\right\|^{2} d t q(d z)<\infty$, a.s. Immediately, from Kuratowski and Ryll-Nardzewski measurable selection theorem (see [3]) it follows that for every $F, \mathcal{G} \in \mathcal{M}_{s-v}^{2}\left(\mathscr{F}_{t}\right)$ and $\mathscr{R}_{\mathcal{B}} \in \mathcal{M}_{s-v}^{2}\left(\mathscr{F}_{t}, q\right)$ their subtrajectory integrals

$$
\begin{gathered}
\varphi(F):=\left\{f \in \mathcal{M}^{2}\left(\mathscr{F}_{t}\right): f_{t}(\omega) \in F_{t}(\omega), d t \times P \text {-a.e. }\right\} \\
\varphi(\mathcal{G}):=\left\{g \in \mathcal{M}^{2}\left(\mathscr{F}_{t}\right): g_{t}(\omega) \in \mathcal{G}_{t}(\omega), d t \times P \text {-a.e. }\right\} \text { and } \\
\Psi_{q}(\mathscr{B}):=\left\{h \in \mathcal{M}^{2}\left(\mathscr{F}_{t}, q\right): h_{t, z}(\omega) \in \mathscr{R}_{t, z}(\omega), d t \times P \times q \text {-a.e. }\right\}
\end{gathered}
$$

are nonempty. Indeed, let $\Sigma=\left\{Z \in \mathscr{B}_{+} \otimes \mathscr{F}_{:} Z_{t} \in \mathscr{F}_{t}\right.$, each $\left.t \geq 0\right\}$, where $Z_{t}$ denotes a section of $Z$ determined by $t \geq 0$. It is a $\sigma$-algebra on $\mathbb{R}_{+} \times \Omega$ and a function $f: \mathbb{R}_{+} \times \Omega \rightarrow \mathbb{R}^{n}$ (a multifunction $\left.F: \mathbb{R}_{+} \times \Omega \rightarrow C l\left(\mathbb{R}^{n}\right)\right)$ is nonanticipative if and only if it is $\Sigma$-measurable. Therefore, 
by Kuratowski and Ryll-Nardzewski measurable selection theorem every nonanticipative setvalued function admits a nonanticipative selector. It is clear that for $F \in \mathcal{M}_{s-v}^{2}\left(\mathcal{F}_{t}\right)$ such selector belongs to $\mathcal{H}^{2}\left(\mathscr{F}_{t}\right)$. Similarly, define on $\mathbb{R}_{+} \times \Omega \times \mathbb{R}^{n}$ a $\sigma$-algebra

$$
\tilde{\Sigma}=\left\{Z \in \mathscr{B}_{+} \otimes \mathscr{F} \otimes \mathscr{B}^{n}: Z_{t}^{u} \in \mathscr{F}_{t} \text {, each } t \geq 0 \text { and } u \in \mathbb{R}^{n}\right\},
$$

where $Z_{t}^{u}=\left(Z^{u}\right)_{t}$ and $Z^{u}$ is a section of $Z$ determined by $u \in \mathbb{R}^{n}$.

Given the set-valued processes

$$
\begin{aligned}
F=\left(F_{t}\right)_{t \geq 0} & \in \mathcal{M}_{s-v}^{2}\left(\mathscr{F}_{t}\right), \mathcal{G}=\left(\mathcal{G}_{t}\right)_{t \geq 0} \in \mathcal{M}_{s-v}^{2}\left(\mathscr{F}_{t}\right) \text { and } \\
\mathscr{R}_{0} & =\left(\mathscr{R}_{t, z}\right)_{t \geq 0, z} \in \mathbb{R}^{n} \in \mathcal{M}_{s-v}^{2}\left(\mathscr{F}_{t}, q\right)
\end{aligned}
$$

by their stochastic integrals we mean families

$$
\left(\int_{0}^{t} F_{\tau} d \tau\right)_{t \geq 0},\left(\int_{0}^{t} \mathfrak{g}_{\tau} d w_{\tau}\right)_{t \geq 0}, \text { and }\left(\int_{0}^{t} \int_{\mathbb{R}^{n}} \mathscr{B}_{\tau, z} \widetilde{\nu}(d \tau, d z)\right)_{t \geq 0}
$$

of subsets defined by

$$
\begin{gathered}
\int_{0}^{t} F_{\tau} d \tau=\left\{\int_{0}^{t} f_{\tau} \tau: f \in \mathcal{Y}(F)\right\}, \\
\int_{0}^{t} g_{\tau} d w_{\tau}=\left\{\int_{0}^{t} g_{\tau} d w_{\tau}: g \in \mathcal{Y}(\mathcal{G})\right\} \text { and } \\
\left\{\int_{0}^{t} \int_{\mathbb{R}^{n}} \mathscr{R}_{\tau, z} \widetilde{\nu}(d \tau, d z)=\left\{\int_{0}^{t} \int_{\mathbb{R}^{n}} h_{\tau, z} \widetilde{\nu}(d \tau, d z): h \in \mathcal{Y}_{q}\left(\mathscr{R}_{0}\right)\right\} .\right.
\end{gathered}
$$

Given $0 \leq \alpha<\beta<\infty$ we also define

$$
\begin{aligned}
& \int_{\alpha}^{\beta} F_{s} d s:=\left\{\int_{\alpha}^{\beta} f_{s} d s: f \in \mathcal{Y}(F)\right\} \\
& \int_{\alpha} \mathfrak{g}_{s} d w_{s}:=\left\{\int_{\alpha}^{\beta} g_{s} d w_{s}: g \in \Psi(\mathcal{g})\right\} \text { and } \\
& \int_{\alpha}^{\beta} \int_{\mathbb{R}^{n}} \mathscr{B}_{s, z} \tilde{\nu}(d s, d z):=\left\{\int_{\alpha}^{\beta} \int_{\mathbb{R}^{n}} h_{s, z} \tilde{\nu}(d s, d z): h \in \Psi_{q}\left(\mathscr{F}^{n}\right)\right\} .
\end{aligned}
$$

\section{Stochastic Inclusions}

Let $F=\left\{\left(F_{t}(x)\right)_{t \geq 0}: x \in \mathbb{R}^{n}\right\}, G=\left\{\left(G_{t}(x)\right)_{t \geq 0}: x \in \mathbb{R}^{n}\right\}$ and $H=\left\{\left(H_{t, z}(x)\right)_{t \geq 0, z \in \mathbb{R}^{n}}\right.$ : $\left.x \in \mathbb{R}^{n}\right\}$. Assume $F, G$ and $H$ are such that $\left(F_{t}(x)\right)_{t \geq 0} \in \mathcal{M}_{s-\nu}^{p}\left(\mathscr{F}_{t}\right),\left(G_{t}(x)\right)_{t \geq 0} \in \mathcal{M}_{s-\nu}^{2}\left(\mathscr{F}_{t}\right)$

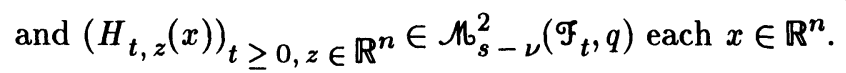

By a stochastic inclusion, denoted by $S I(F, G, H)$, corresponding to given above $F, G$ and $H$ we mean a relation

$$
x_{t}-x_{s} \in \int_{s}^{t} F_{\tau}\left(x_{\tau}\right) d \tau+\int_{s}^{t} G_{\tau}\left(x_{\tau}\right) d w_{\tau}+\int_{s}^{t} \int_{\mathbb{R}} H_{\tau, z}\left(x_{\tau}\right) \tilde{\nu}(d \tau, d z)
$$

that is to be satisfied for every $0 \leq s<t<\infty$ by a stochastic process $x=\left(x_{t}\right)_{t} \geq_{0} \in D$ such that $F \circ x \in \mathcal{M}_{s-\nu}^{p}\left(\mathcal{F}_{t}\right), G \circ x \in \mathcal{M}_{s-\nu}^{2}\left(\mathscr{F}_{t}\right)$ and $H \circ x \in \mathcal{H}_{s-\nu}^{2}\left(\mathscr{F}_{t}, q\right)$, where $F \circ x=\left(F_{t}\left(x_{t}\right)\right)_{t \geq 0}$,

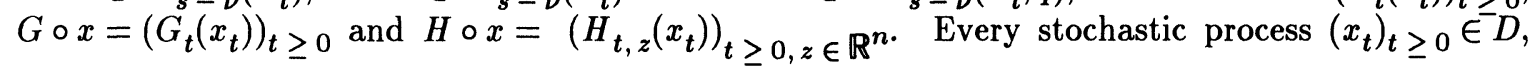
satisfying conditions mentioned above is said to be a global solution to $S I(F, G, H)$. 
A stochastic process $\left(x_{t}\right)_{t \geq 0} \in D$ is a local solution to $S I(F, G, H)$ on $[\alpha, \beta]$ if and only if $x^{\alpha, \beta}$ is a global solution to $S I\left(F^{\alpha \beta}, G^{\alpha \beta}, H^{\alpha \beta}\right)$, where $F^{\alpha \beta}=\rrbracket_{[\alpha, b]} F, \quad G^{\alpha \beta}=\rrbracket_{[\alpha, \beta]} G$ and

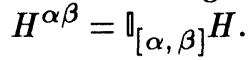

A stochastic process $\left(x_{t}\right)_{t>0} \in D$ is called a global (local on $[\alpha, \beta]$, resp.) solution to an initial value problem for stochastic inclusion $S I(F, G, H)$ with an initial condition $y \in L^{2}\left(\Omega, \mathcal{F}_{0}, \mathbb{R}^{n}\right)$ $\left(y \in \mathscr{F}_{\alpha}, \mathbb{R}^{n}\right)$, resp.) if $\left(x_{t}\right)_{t \geq 0}$ is a global (local on $[\alpha, \beta]$, resp.) solution to $S I(F, G<h)$ and $x_{0}=y\left(x_{\alpha}=y\right.$, resp.). An initial-value problem for $S I(F, G, H)$ mentioned above will be denoted by $S I_{y}(F, G, H)\left(S_{y}^{\alpha, \beta}(F, G, H)\right.$, resp.). In what follows, we denote a set of all global (local on $[\alpha, \beta]$ solutions to $S I_{y}(F, G, H)$ by $\Lambda_{y}(F, G, H)\left(\Lambda_{y}^{\alpha, \beta}(F, G, H)\right.$, resp. $)$.

Suppose $F, G$ and $H$ satisfy the following conditions:

$\left(\mathcal{A}_{1}\right) \quad(i) \quad F=\left\{\left(F_{t}(x)\right)_{t \geq 0}: x \in \mathbb{R}^{n}\right\}, G=\left\{\left(G_{t}(x)\right)_{t>0}: x \in \mathbb{R}^{n}\right\}$ and $H=$

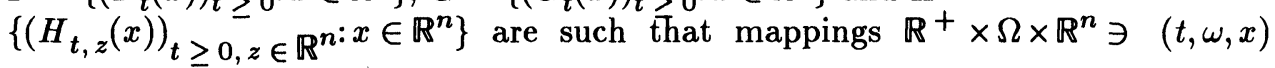
$\rightarrow F_{t}(x)(\omega) \in \operatorname{Comp}\left(\mathbb{R}^{n}\right), \quad \mathbb{R}_{+} \times \Omega \times \mathbb{R}^{n} \ni(t, \omega, x) \rightarrow G_{t}(x)(\omega) \in \quad \operatorname{Comp}\left(\mathbb{R}_{\sim}^{n}\right)$ and $\mathbb{R}_{+} \times \Omega \times \mathbb{R}^{n} \times \mathbb{R}^{n} \ni(t, \omega, z, x) \rightarrow H_{t, z}(x)(\omega) \in \operatorname{Comp}\left(\mathbb{R}^{n}\right)$ are $\Sigma \otimes \mathfrak{B}^{n}$ and $\widetilde{\Sigma} \otimes \mathfrak{B}^{n}-$ measurable, respectively, where $\Sigma$ and $\tilde{\Sigma}$ are $\sigma$-algebras on $\mathbb{R}_{+} \times \Omega$ and $\mathbb{R}_{+} \times \Omega \times \mathbb{R}^{n}$ defined above,

(ii) $\quad\left(F_{t}(x)\right)_{t \geq 0},\left(G_{t}(x)\right)_{t \geq 0}$ and $\left(H_{x, z}(x)\right)_{t \geq 0, z \in \mathbb{R}^{n}}$ are square integrable bounded for fixed $x \in \mathbb{R}^{n}$.

Corollary 1: For every $\left(x_{t}\right)_{t>0} \in D$ and $F, G, H$ satisfying $\left(\mathcal{A}_{1}\right)$ one has $F \circ x$, $G \circ x \in \mathcal{M}_{s-\nu}^{2}\left(\mathscr{F}_{t}\right)$ and $H \circ x \in \mathcal{M}_{s-\nu}^{2}\left(\mathscr{F}_{t}, q\right)$.

Now, define a linear mapping $\Phi$ on $\mathcal{M}^{2}\left(\mathscr{F}_{t}\right) \times \mathcal{H}^{2}\left(\mathscr{F}_{t} \times \mathcal{M}^{2}\left(\mathscr{F}_{t}, q\right)\right.$ by taking $\Phi(f, g, h)$ $\left.=\int_{0}^{t} f_{\tau} d \tau+\int_{0}^{t} g_{\tau} d w_{\tau}+\int_{0}^{t} \int_{\mathbb{R}^{n}} h_{\tau, z} \widetilde{\nu}(d \tau, d z)\right)_{t \geq 0}$ to each $(f, g, h) \in \mathcal{M}^{2}\left(\mathscr{F}_{t}\right) \times \mathcal{H}^{2}\left(\mathscr{F}_{t}\right) \times \mathcal{M}^{2}\left(\mathscr{F}_{t}, q\right)$.

It is clear that $\Phi$ maps $\ell_{n}^{2} \times \mathcal{L}_{n}^{2} \times W_{n}^{2}$ into $D$.

In what follows, we shall deal with $F=\left\{\left(F_{t}(x)\right)_{t>0}: x \in \mathbb{R}^{n}\right\}, G=\left\{\left(G_{t}(x)\right)_{t>0}: x \in \mathbb{R}^{n}\right\}$

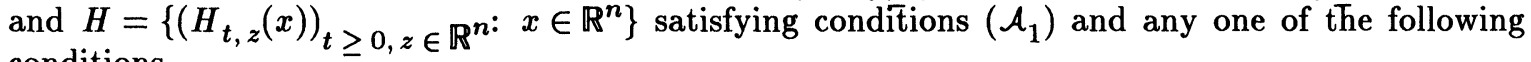
conditions.

$\left(\mathcal{A}_{2}\right)$ There are $k, \ell \in \mathcal{L}_{1}^{2}$ and $m \in \mathcal{W}_{1}^{2}$ such that $\left\|\int_{0}^{\infty} h\left[(F \circ x)_{t}, \quad(F \circ y)_{t}\right] d t\right\|_{\mathcal{L}_{1}^{2}} \leq$ $E \int_{0}^{\infty} k_{t}\left|x_{t}-y_{t}\right| d t, \quad\|h(G \circ x, G \circ y)\|_{\ell_{1}^{2}} \leq E \int_{0}^{\infty} \ell_{t}\left|x_{t}-y_{t}\right| d t \quad$ and $\quad \| h(H \circ x$ $H \circ y) \|_{W_{1}^{2}} \leq E \int_{0}^{\infty} \int_{\mathbb{R}^{n}} m_{t, z}\left|x_{t}-y_{t}\right| d t q(d z)$ for all $x, y \in D$.

$\left(\mathcal{A}_{3}\right)$ There are $k, \ell \in L^{2}\left(\mathscr{B}_{+}\right)$and $m \in L^{2}\left(\mathscr{B}_{+} \times \mathfrak{B}^{n}\right)$ such that $h\left(F_{t}\left(x_{2}\right)(\omega)\right.$, $\left.F_{t}\left(x_{1}\right)(\omega)\right) \leq k(t)\left|x_{1}-x_{2}\right|, \quad h\left(G_{t}\left(x_{2}\right)(\omega), G_{t}\left(x_{1}\right)(\omega)\right) \leq \ell(t)\left|x_{1}-x_{2}\right| \quad$ and $h\left(H_{t, z}\left(x_{2}\right)(\omega), H_{t, z}\left(x_{1}\right)(\omega)\right) \leq m(t, z)\left|x_{1}-x_{2}\right|$ a.e., each $t \geq 0$ and $x_{1}, x_{2} \in \mathbb{R}^{n}$.

Lemma 1: Let $\varphi \in L^{2}\left(\Omega, \mathscr{F}_{0}, \mathbb{R}^{n}\right)$. Suppose $F, G$ and $H$ satisfy $\left(\mathcal{A}_{1}\right)$ and $\left(\mathcal{A}_{2}\right)$ or $\left(\mathcal{A}_{3}\right)$. Let $x^{n}=\varphi+\Phi\left(f^{n-1}, g^{n-1}, h^{n-1}\right)$, each $n=1,2, \ldots$, with $\left(f^{0}, g^{0}, h^{0}\right) \in \varphi(F \circ 0) \times \varphi(G \circ 0) \times \varphi_{q}(H \circ 0)$ and $\quad\left(f^{n}, g^{n}, h^{n}\right) \in \varphi\left(F \circ x^{n}\right) \times \varphi\left(G \circ x^{n}\right) \times \varphi_{q}\left(H \circ x^{n}\right) \quad$ satisfying $\quad\left|f_{t}^{n-1}(\omega)-f_{t}^{n}(\omega)\right|$ $=\operatorname{dist}\left(f_{t}^{n-1}(\omega),\left(F \circ x^{n}\right)_{t}(\omega)\right), \quad\left|g_{t}^{n-1}(\omega)-g_{t}^{n}(\omega)\right|=\operatorname{dist}\left(g_{t}^{n-1}(\omega),\left(G \circ x^{n}\right)_{t}(\omega)\right) \quad$ and $\left|h_{t, z}^{n-1}(\omega)-h_{t, z}^{n}(\omega)\right|=\operatorname{dist}\left(h_{t, z}^{n-1}(\omega),\left(H \circ x^{n}\right)_{t, z}(\omega)\right), \quad$ on $\quad \mathbb{R}_{+} \times \Omega \quad$ and $\quad \mathbb{R}_{+} \times \Omega \times \mathbb{R}^{n}$, respectively. If $L:=\left\|\int_{0}^{\infty} k_{t} d t\right\|_{L_{1}^{2}}+2\left\|\int_{0}^{\infty} \ell_{t} d t\right\|_{L_{1}^{2}}+2\left\|\int_{0}^{\infty} \int_{\mathbb{R}^{n} m_{\tau, z}} d \tau q(d z)\right\|_{L_{1}^{2}}<1 \quad$ or $L^{\prime}:=|k|_{1}+2|\ell|_{2}+2\|m\|_{2}<1$, respectively then $\left(x^{n}\right)_{n=1}^{\infty}$ is a Cauchy sequence of $\left(D,\|\cdot\|_{\ell}\right)$. 
Proof: Let $\left(x^{n}\right)_{n=1}^{\infty}$ be such as above. By $\left(\mathcal{A}_{2}\right)$ it follows

$$
\begin{aligned}
& E\left[\sup _{t \geq 0}\left|\int_{0}^{t}\left(f_{\tau}^{n}-f_{\tau}^{n-1}\right) d \tau\right|\right]^{2} \leq E\left[\int_{0}^{\infty}\left|f_{\tau}^{n}-f_{\tau}^{n-1}\right| d \tau\right]^{2} \\
& \leq E\left[\int_{0}^{\infty} \bar{h}\left(\left(F \circ x^{n}\right)_{\tau},\left(F \circ x^{n-1}\right)_{\tau}\right) d \tau\right]^{2} \leq\left(E \int_{0}^{\infty} k_{\tau}\left|x_{\tau}^{n}-x_{\tau}^{n-1}\right| d \tau\right)^{2} \\
& \leq\left[E\left(\sup _{t \geq 0}\left|x_{t}^{n}-x_{t}^{n-1}\right| \cdot \int_{0}^{\infty} k_{\tau} d \tau\right)\right]^{2} \leq E\left(\int_{0}^{\infty} k_{\tau} d \tau\right)^{2} \cdot\left\|x^{n}-x^{n-1}\right\|_{\ell}^{2} .
\end{aligned}
$$

Similarly, by Doob's inequality, we obtain

$$
\begin{gathered}
\left.E\left[\sup _{t \geq 0} \mid \int_{0}^{t}\left(g_{\tau}^{n}-g_{\tau}^{n-1}\right) d w_{\tau}\right]\right]^{2} \leq 4 E \int_{0}^{\infty}\left|g_{\tau}^{n}-g_{\tau}^{n-1}\right|^{2} d \tau \\
\leq 4 E \int_{0}^{\infty}\left[\bar{h}\left(\left(G \circ x^{n}\right)_{\tau},\left(G \circ x^{n-1}\right)_{\tau}\right)\right]^{2} d \tau \leq 4\left(E \int_{0}^{\infty} \ell_{\tau}\left|x_{\tau}^{n}-x_{\tau}^{n-1}\right| d \tau\right)^{2} \\
\leq 4\left[E\left(\sup _{t \geq 0}\left|x_{t}^{n}-x_{t}^{n-1}\right| \cdot \int_{0}^{\infty} \ell_{\tau} d \tau\right)\right]^{2} \leq 4 E\left(\int_{0}^{\infty} \ell_{\tau} d \tau\right)^{2} \cdot\left\|x^{n}-x^{n-1}\right\|_{\ell}^{2} .
\end{gathered}
$$

Quite similarly we also get

$$
\begin{aligned}
& \left.E\left[\sup _{t \geq 0} \mid \int_{0}^{t} \int_{\mathbb{R}^{n}}\left(h_{\tau}^{n}-h_{\tau, z}^{n-1}\right) \tilde{\nu}(d \tau, d z)\right]\right]^{2} \\
& \leq 4 E\left(\int_{0}^{\infty} \int_{\mathbb{R}^{n}} m_{\tau, z} d \tau q(d z)\right)^{2} \cdot\left\|x_{\tau}^{n}-x_{\tau}^{n-1}\right\|_{\ell}^{2} .
\end{aligned}
$$

Therefore, $\left\|x^{n+1}-x^{n}\right\|_{\ell} \leq L^{n}\left\|x^{1}\right\|_{\ell}$, where $L$ is such as above. This implies that

$$
\left\|x^{m}-x^{n}\right\|_{\ell} \leq \frac{L^{n} \cdot\left\|x^{1}\right\|_{\ell}}{1-L}
$$

each $m>n \geq 1$. Using conditions $\left(\mathcal{A}_{3}\right)$ instead of $\left(\mathcal{A}_{2}\right)$ we also get

$$
\left\|x^{m}-x^{n}\right\|_{\ell} \leq \frac{\left(L^{\prime}\right)^{n} \cdot\left\|x^{1}\right\|_{\ell}}{1-L^{\prime}}
$$

for $m>n \geq 1$. Therefore, $\left\|x^{m}-x^{n}\right\|_{\ell \rightarrow 0}$ as $n \rightarrow \infty$. 
Lemma 2: Let $\varphi \in L^{2}\left(\Omega, \mathscr{F}_{0}, \mathbb{R}^{n}\right)$. Suppose $F, G$ and $H$ satisfy $\left(\mathcal{A}_{1}\right)$ and $\left(\mathcal{A}_{3}\right)$. IF $L:=|k|_{1}+2|\ell|_{2}+2\|m\|_{2}<1$, then $\Lambda_{\varphi}(F, G, H) \neq \emptyset$.

Proof: Let $\left(x^{n}\right)_{n=1}^{\infty}$ be such as in Lemma 1 and let $x=\lim _{n \rightarrow \infty} x^{n}$. The existence of such a sequence follows immediately from the measurable selection theorem given in [3] (see Th. II, 3.13). We shall now show that $\left(f^{n}\right)_{n=1}^{\infty},\left(g^{n}\right)_{n=1}^{\infty}$ and $\left(h^{n}\right)_{n=1}^{\infty}$ are Cauchy sequences of $\mathcal{L}_{n}^{2}$ and $W_{n}^{2}$, respectively. Indeed, one obtains

$$
\begin{gathered}
\left\|f^{m}-f^{n}\right\|_{\ell_{n}^{2}}=\sum_{j=n+1}^{m}\left[\left\|f^{j}-f^{j-1}\right\|_{\ell_{n}^{2}}\right]^{1 / 2} \\
\leq \sum_{j=n+1}^{m}\left[E \int_{0}^{\infty} h^{2}\left(\left(F \circ x^{j}\right)_{\tau},\left(F \circ x^{j-1}\right)_{\tau}\right) d \tau\right]^{1 / 2} \\
\leq \sum_{j=n+1}^{m}|k|_{2}\left\|x^{j}-x^{j-1}\right\|_{\ell} \leq \sum_{j=n+1}^{m} L^{j-1}|k|_{2}\left\|x^{1}\right\|_{\ell} \leq \frac{L^{n}|k|_{2}\left\|x^{1}\right\|_{\ell}}{1-L} .
\end{gathered}
$$

Therefore, $\left(f^{n}\right)_{n=1}^{\infty}$ is a Cauchy sequence of $\ell_{n}^{2}$. Quite similarly, it also follows that $\left(g^{n}\right)_{n=1}^{\infty}$ and $\left(h^{n}\right)_{n=1}^{\infty}$ are Cauchy sequences of $\mathcal{L}_{n}^{2}$ and $W_{n}^{2}$, respectively. Let $f, g \in \mathcal{L}_{n}^{2}$ and $h \in W_{n}^{2}$ be such that $\left\|f^{n}-f\right\|_{\mathcal{L}_{n}^{2} \rightarrow 0,}\left\|g^{n}-g\right\|_{\mathcal{L}_{n}^{2} \rightarrow 0}$ and $\left|h^{n}-h\right|_{W_{n}^{2} \rightarrow 0}$ as $n \rightarrow \infty$. One gets $\left\|x^{n}-\varphi-\Phi(f, g, h)\right\|_{\ell \rightarrow 0}$ as $n \rightarrow \infty$. Therefore, $x=\varphi+\Phi(f, g, h)$. To prove that

$$
x_{t}-x_{s} \in \int_{s}^{t}(F \circ x)_{\tau} d \tau+\int_{s}^{t}(G \circ x)_{\tau} d w_{\tau}+\int_{s}^{t} \int_{\mathbb{R}^{n}}(H \circ x)_{\tau, z} \tilde{\nu}(d \tau, d z)
$$

for every $0 \leq s<t<\infty$ it suffices only to verify that $(f, g, h) \in \varphi(F \circ x) \times \varphi(G \circ x) \times \varphi_{g}(H \circ x)$. For this aim, denote by $\operatorname{Dist}(a, B)$ and $\bar{H}$ the distance of $a \in \mathcal{L}_{n}^{2}$ to a nonempty set $B \subset \mathcal{L}_{n}^{2}$ and the Hausdorff subdistance, respectively induced by the norm of $\ell_{n}^{2}$. Now let $v$ be a fixed element of $\varphi\left(F \circ x^{n}\right)$. Select $u \in \varphi(F \circ x)$ such that $\left|v_{\tau}(\omega)-u_{\tau}(\omega)\right|=\operatorname{dist}\left(v_{\tau}(\omega),(F \circ x)_{\tau}(\omega)\right)$ for $(\tau, \omega) \in \mathbb{R}_{+} \times \Omega$. Then

$$
\begin{gathered}
\operatorname{Dist}(v, \Psi(F \circ x)) \leq\|v-u\|_{\mathcal{L}_{n}^{2}} \\
\left.\leq\left(E \int_{0}^{\infty} h^{2}\left(\left(F \circ x^{n}\right)_{\tau}(\omega)\right),(F \circ x)_{\tau}(\omega)\right) d \tau\right)^{\frac{1}{2}} \leq|k|_{2}\left\|x^{n}-x\right\|_{\ell^{\prime}}
\end{gathered}
$$

which implies $\bar{H}\left(\varphi\left(F \circ x^{n}\right), \varphi(F \circ x)\right) \leq|k|_{2}\left\|x^{n}-x\right\|_{\ell}$, each $n=1,2, \ldots$ Thus $\bar{H}\left(\varphi\left(F \circ x^{n}\right)\right.$, $\varphi(F \circ p x)) \rightarrow 0$ as $n \rightarrow \infty$. In a similar way we also get $\bar{H}\left(\varphi\left(G \circ x^{n}\right), \quad \varphi(G \circ x) \rightarrow 0\right.$ and $\bar{H}\left(\varphi_{q}\left(H \circ x^{n}\right), \varphi_{q}(H \circ x)\right) \rightarrow 0$ as $n \rightarrow \infty$. Now we get

$$
\begin{gathered}
\operatorname{Dist}(f, \varphi(F \circ x)) \leq\left\|f-f_{n}\right\|_{\mathcal{L}_{n}^{2}}+\operatorname{Dist}\left(f_{n}, \varphi\left(F \circ x^{n-1}\right)\right) \\
+\bar{H}\left(\varphi\left(F \circ x^{n-1}\right), \varphi(F \circ x)\right)
\end{gathered}
$$

for $n=1,2, \ldots$, which implies that $\operatorname{Dist}(f, \varphi(F \circ x))=0$. But, $\varphi(F \circ x)$ is a nonempty closed subset of $\mathcal{L}_{n}^{2}$. Therefore, $f \in \varphi(F \circ x)$. In a similar way we can also verify that $g \in \varphi(G \circ x)$ and $h \in \varphi_{q}(H \circ x)$. 
Lemma 3: Let $0 \leq \alpha<\beta<\infty$ and $\varphi \in L^{2}\left(\Omega, \mathscr{F}_{\alpha}, \mathbb{R}^{n}\right)$. Suppose $F$, $G$, and $H$ satisfy $\left(\mathcal{A}_{1}\right)$ and $\left(\mathcal{A}_{3}\right)$. If $L_{\alpha, \beta}:=\left|\mathbb{q}_{[\alpha, \beta]} k\right|_{1}+2\left|q_{[\alpha, \beta]} \ell\right|_{2}+2\left\|\nabla_{[\alpha, \beta]} m\right\|_{2}<1$ then $\Lambda_{\varphi}^{\alpha, \beta}(F, G, H) \neq \emptyset$.

Proof: The proof follows immediately from Lemma 2 applied to $F^{\alpha \beta}=\mathbb{\square}_{[\alpha, \beta]} F$, $G^{\alpha \beta}=\mathbb{\square}_{[\alpha, \beta} G$ and $H^{\alpha \beta}=\rrbracket_{[\alpha, \beta]} H$.

Lemma 4: Let $\varphi \in L^{2}\left(\Omega, \mathscr{F}_{0}, \mathbb{R}^{n}\right)$ and let $\left(\tau_{n}\right)_{n=1}^{\infty}$ be a sequence of positive number increasing to $+\infty$. Suppose $F, G$ and $H$ satisfy $\left(\mathcal{A}_{1}\right)$ and $\left(\mathcal{A}_{3}\right)$. If $x^{1} \in \Lambda_{\varphi}^{0, \tau_{1}}(F, G, H)$ and

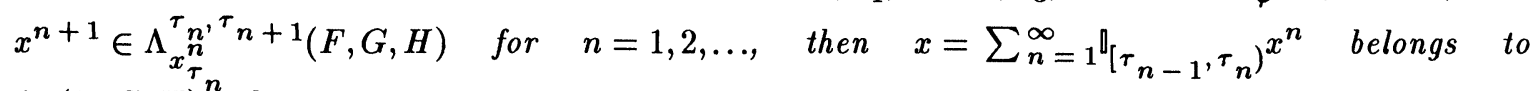
$\Lambda_{\varphi}(F, G, H)$, where $\tau_{0}=0$.

Proof: It is clear that $x_{0}=\varphi$ because $x_{0}=x_{0}^{1}=\varphi$. Let $0 \leq s<t<\infty$ be fixed and suppose $s \in\left[\tau_{k-1}, \tau_{k}\right)$, and $t \in\left[\tau_{m-1}, \tau_{m}\right)$, for $1 \leq k<m$. One obtains

$$
x_{t}-x_{s}=\left(x_{t}^{m}-x_{\tau_{m-1}}^{m}\right)+\left(x_{\tau_{m-1}-1}^{m-x_{\tau_{m-2}}^{m-1}}\right)+\ldots+\left(x_{\tau_{k=1}+1}^{k+1}-x_{\tau_{k}}^{k+1}\right)+\left(x_{\tau_{k}}^{k}-x_{s}^{k}\right) .
$$

Let $\left(f^{j}, g^{j}, h^{j}\right) \in S\left(F \circ x^{j}\right) \times S\left(G \circ x^{j}\right) \times S_{q}\left(H \circ x^{j}\right)$, each $j=k, k+1, \ldots, m$ be such that

$$
\begin{gathered}
x_{t}^{m}-x_{\tau_{m-1}}^{m}=\int_{\tau_{m-1}^{t}}^{t} f_{\tau}^{m} d \tau+\int_{\tau_{m-1}}^{t} g_{\tau}^{m} d w_{\tau}+\int_{\tau_{m-1}}^{t} \int_{\mathbb{R}^{n}} h_{\tau}^{m} \tilde{\nu}(d \tau, d z), \\
x_{\tau_{j}-x_{\tau_{j-1}}^{j}}=\int_{\tau-1}^{\tau_{j}} f_{\tau}^{j} d \tau+\int_{\tau_{j-1}}^{\tau_{j}} g_{\tau}^{j} d w_{\tau}+\int_{\tau}^{j} \int_{j-1 \mathbb{R}^{n}} h_{\tau}^{j} \widetilde{\nu}(d t, d z),
\end{gathered}
$$

each $j=k+1, \ldots, m-1$, and

$$
x_{\tau_{k}}^{k}-x_{s}^{k}=\int_{s}^{\tau_{k}} f_{\tau}^{k} d \tau+\int_{s}^{\tau_{k}} g_{\tau}^{k} d w_{\tau}+\int_{s}^{\tau_{k}} \int_{\mathbb{R}^{n}} h_{\tau}^{k} \tilde{\nu}(d \tau, d z)
$$

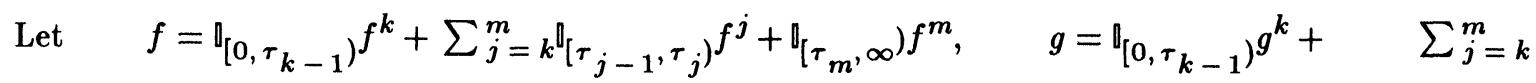

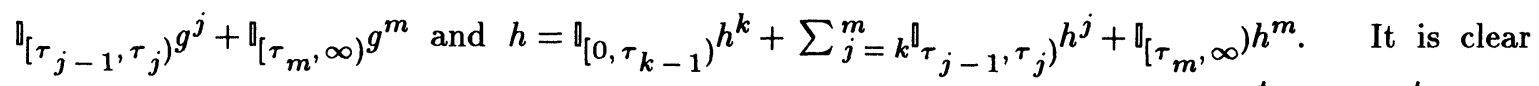
that $\quad(f, g, h) \in S(F \circ x) \times S(G \circ c) \times S_{q}(H \circ x) \quad$ and $\quad x_{t}-x_{s}=\int_{s}^{t} f_{\tau} d \tau+\int_{s}^{t} g_{\tau} d w_{\tau}$ $+\int_{s}^{t} \int_{\mathbb{R}^{n}} h_{\tau, z} \widetilde{\nu}(d \tau, d z)$. Therefore

$$
x_{t}-x_{s} \in \int_{s}^{t}(F \circ x)_{\tau} d \tau+\int_{s}^{t}(G \circ x)_{\tau} d w_{\tau}+\int_{s}^{t} \int_{\mathbb{R}^{n}}(H \circ x)_{\tau, z} \widetilde{\nu}(d \tau, d z) .
$$

We can prove now the main result of this paper.

Theorem 5: Let $\varphi \in L^{2}\left(\Omega, \mathscr{F}_{0}, \mathbb{R}^{n}\right)$. Suppose $F, G$ and $H$ satisfy $\left(\mathcal{A}_{1}\right)$ and $\left(\mathcal{A}_{3}\right)$. Then $\Lambda_{\varphi}(F, G, H) \neq \emptyset$.

Proof: Let $\left(\tau_{n}\right)_{n=1}^{\infty}$ be a sequence of positive numbers increasing to $\infty$. Select a positive number $\sigma$ such that $L_{k \sigma,(k 1) \sigma}<1$ for $k=0,1, \ldots$, where $L_{k \sigma,(k+1) \sigma}$ is such as in Lemma 3 . Suppose a positive integer $n_{1}$ is such that $n_{1} \sigma<\tau_{1} \leq\left(n_{1}+1\right) \sigma$. By virtue of Lemma 3, there 
is $z^{1} \in \Lambda_{\varphi}^{0, \sigma}(F, G, H)$. By the same argument, there is $z^{2} \in \Lambda_{z_{\sigma}}^{\sigma}{ }_{i}^{2 \sigma}(F, G, H)$. Continuing the above procedure we can finally finc a $z^{n_{1}+1} \in \Lambda_{z_{n_{1} \sigma}^{n_{1} \sigma, n_{1}}}^{n_{1}}(F, G, H)$. $\stackrel{z_{\sigma}}{\mathrm{P} u t}$

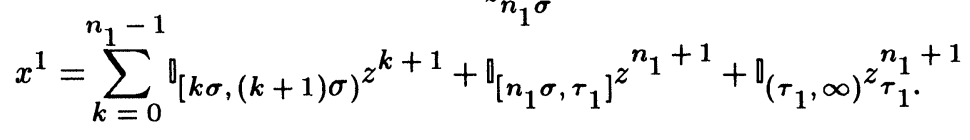

Similarly as in the proof of Lemma 4, we can easily verity that $x^{1} \in \Lambda_{\varphi}^{0, \tau} 1(F, G, H)$. Repeating the above procedure to the interval $\left[\tau_{1}, \tau_{2}\right]$, we can find $x^{2} \in \Lambda_{x_{\tau_{1}}}^{\tau_{1}, \tau_{2}}(F, G, H)$. Continuing this process, we can define a sequence $\left(x^{n}\right)$ of $D$ satisfying conditions of Lemma 4 . Therefore $\Lambda_{\varphi}(F, G, H) \neq \emptyset$.

\section{REFERENCES}

[1] Gihman, I.I., Skorohod, A.V., Controlled Stochastic Processes, Springer-Verlag, Berlin, New York 1979.

[2] Hiai, F. and Umegaki, H., Integrals, conditional expectations and martingales of multi functions, J. Multivariate Anal. 7 (1977), 149-182.

[3] Kisielewicz, M., Differential Inclusions and Optimal Control, Kluwer Acad. Publ. and Polish Sci. Publ., Warszawa-Dordrecht-Boston-London 1991.

[4] Kisielewicz, M., Properties of solution sets of stochastic inclusions, Journal of Appl. Math. and Stochastic Anal. 6:3 (1993), 217-236.

[5] Kisielewicz, M., Set-valued stochastic integrals and stochastic inclusions, Stochastic Anal. and Appl., (to appear).

[6] Protter, Ph., Stochastic Integration and Differential Equations, Springer-Verlag, BerlinHeidelberg-New York 1990. 


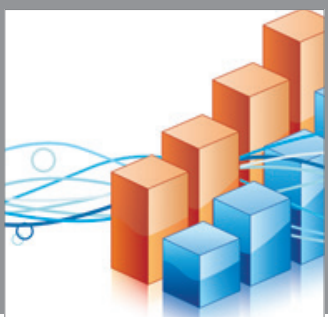

Advances in

Operations Research

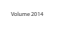

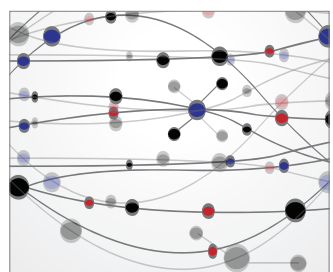

\section{The Scientific} World Journal
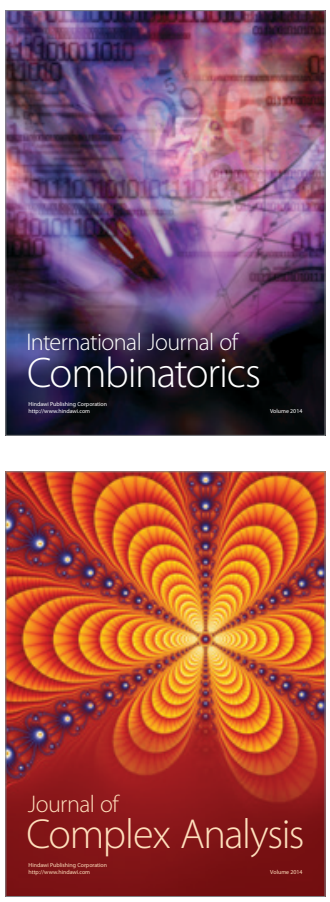

International Journal of

Mathematics and

Mathematical

Sciences
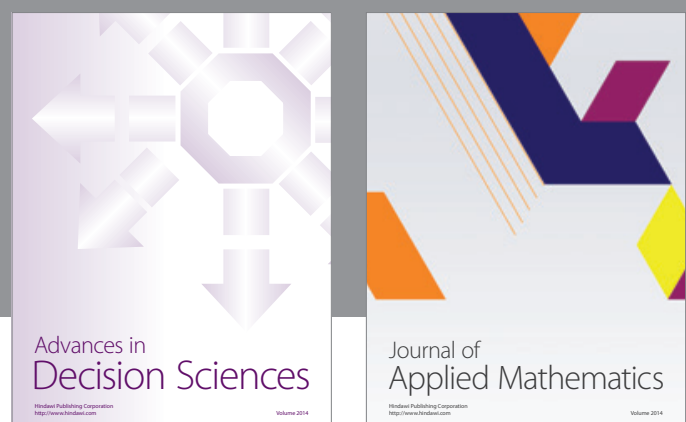

Journal of

Applied Mathematics
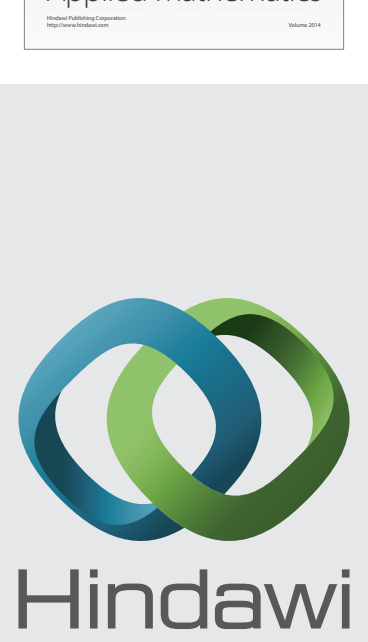

Submit your manuscripts at http://www.hindawi.com
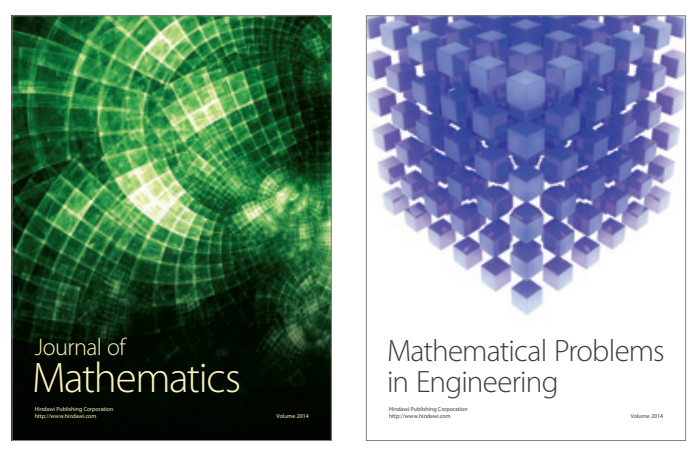

Mathematical Problems in Engineering
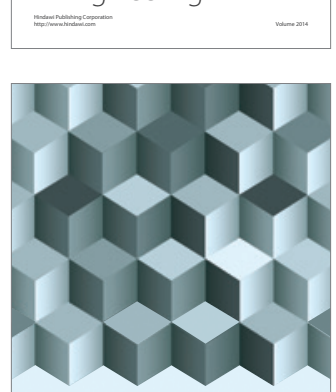

Journal of

Function Spaces
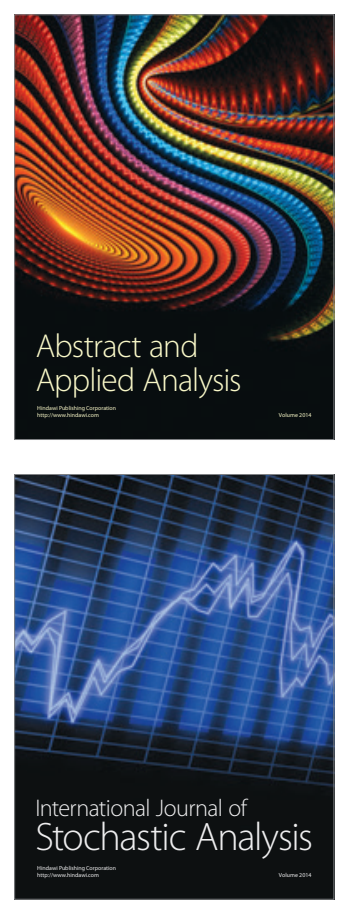

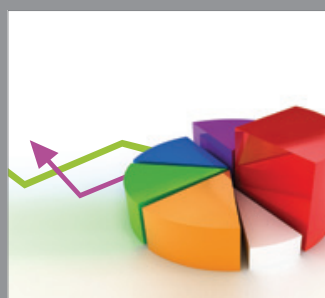

ournal of

Probability and Statistics

Promensencen
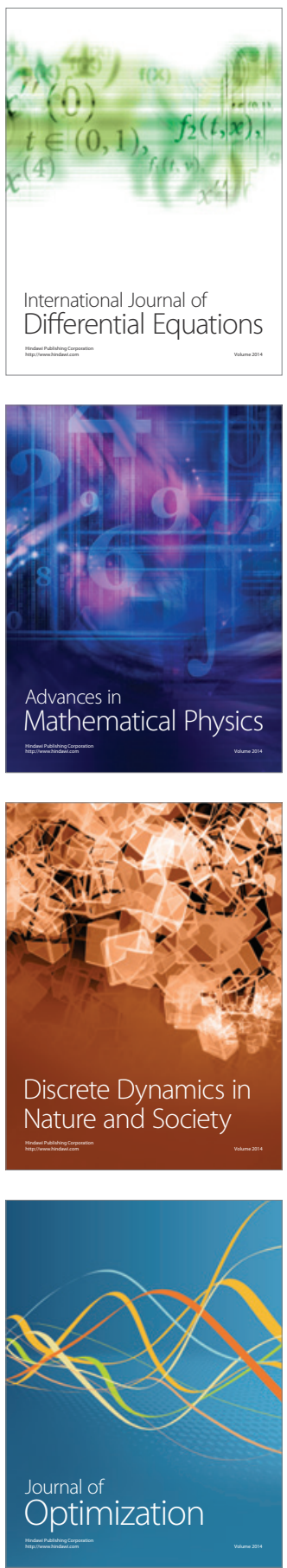